\title{
The Use of Polysiloxane/polyvinyl Alcohol Beads as Solid Phase in IgG Anti-Toxocara canis Detection Using a Recombinant Antigen
}

\author{
Raquel de Andrade Lima Coêlho, Hiroshi Yamasaki*, Emília Perez**, \\ Luiz Bezerra de Carvalho Jr/ ${ }^{+}$
}

\begin{abstract}
Laboratório de Imunopatologia Keizo Asami and Departamento de Bioquímica, Universidade Federal de Pernambuco, Campus Universitário, 50670-901 Recife, PE, Brasil *Department of Parasitology, Asahikawa Medical Colege, Asahikawa, Japan **Departamento Materno Infantil, Universidade Federal de Pernambuco, Recife, PE, Brasil.
\end{abstract}

Immunodetection of human IgG anti-Toxocara canis was developed based on ELISA and on the use of polysiloxane/ polyvinyl alcohol (POS/PVA) beads. A recombinant antigen was covalently immobilized, via glutaraldehyde, onto this hybrid inorganic-organic composite, which was prepared by the sol-gel technique. Using only $31.2 \mathrm{ng}$ antigen per bead, a peroxidase conjugate dilution of 1:10,000 and a serum dilution of 1:200 were adequate for the establishment of the procedure. This procedure is comparable to that which utilizes the adsorption of the antigen to conventional PVC plates. However, the difference between positive and negative sera mean absorbances was larger for this new glass based assay. In addition to the performance of the POS/PVA bead as a matrix for immunodetection, its easy synthesis and low cost are additional advantages for commercial application.

Key words: polysiloxane - polyvinyl alcohol - recombinant antigen - Toxocara canis

Toxocariasis is a helmithozoonosis caused by ascarid larvae belonging to the genus Toxocara (Magnaval et al. 2001). Most frequently caused by T. canis, the prolonged migration of the larvae into the body results in visceral larva migrans syndrome (VLM), affecting mainly children (Lescano et al. 1998).

The diagnosis and confirmation of human toxocariasis rely heavily upon immunological examinations because of the difficulty in detecting infective larvae in biopsy samples (Ajayi et al. 2000, Yamasaki et al. 2000). Therefore, ELISA and Western-blotting using excretory-secretory antigens from $T$. canis second-stage larva (TES) compose the basis of this disease's diagnosis (Magnaval et al. 2001).

Recently, a recombinant $T$. canis second-stage larva antigen (rTc), corresponding to the $30 \mathrm{kDa}$ protein from TES secreted by infective larvae was developed and its sensitivity and specificity tested (Yamasaki et al. 1998, 2000). When the TES was used at concentrations of 0.5 $\mu \mathrm{g} / \mathrm{ml}$, cross-reactions were observed in $79(55.6 \%)$ of 142 cases. In contrast, when the rTc was tested at $0.5 \mu \mathrm{g} / \mathrm{ml}$, cross-reactions were observed in $19(13.4 \%)$, and at 0.125 $\mu \mathrm{g} / \mathrm{ml}$ decreased sharply to only $2.1 \%$, corresponding to 3 of 142 cases (gnathostomiasis, Paragonimus miyazakii and spirometriasis). No cross-reaction was detected with serum from patients infected with Ascaris and hookworms.

In our laboratories silica glass beads were synthesized by alkoxide sol-gel process and were used as solid phase in ELISA for experimental plague studies (Barros et al. 2002). In this procedure, two steps are involved: (a) the hydrolysis of alkoxysilanes precursors and (b) subsequent

Corresponding author. Fax: +55-81-3271.8484. E-mail: lbcj@hotlink.com.br

Received 3 May 2002

Accepted 23 January 2003 condensation of the remaining silanols, followed by aging and drying under ambient atmospheres. They can be summarized as

(a)

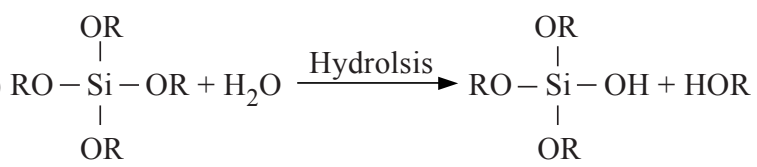

(b) 2

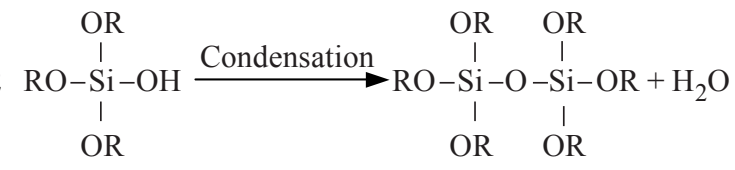

Furthermore, these beads were prepared as an hybrid material provided polyvinyl alcohol (PVA) was incorporated into the glass. The resulting composite combined the PVA property to covalently retain antigens, via glutaraldehyde, with excellent optical, thermal, and chemical stability of the host silicon oxide matrix.

The purpose of the present paper is to report the use of the recombinant antigen covalently immobilized onto polysiloxane/polyvinyl alcohol (POS/PVA) beads in an enzyme immunoassay for the detection of human anti- $T$. canis IgG.

\section{MATERIALS AND METHODS}

Antigen and sera - Recombinant T. canis antigen was obtained according to Yamasaki et al. (2000), with a final protein concentration of $140 \mu \mathrm{g}$ of protein $/ \mathrm{ml}$. The sera samples used throughout this work were those from 45 children attending a daycare institution in Jaboatão, PE, Brazil. Previously, they were clinically examined and their sera tested for toxocariasis (Yamasaki et al. 2000) and divided into a negative ( 24 children) and a positive ( 21 children) group.

Solid support synthesis - POS/PVA beads were synthesized according to Barros et al. (2002). Briefly: $6 \mathrm{ml}$ of 
$2 \% \mathrm{w} / \mathrm{v}$ polyvinyl alcohol (MW 72000; Reagen), $5 \mathrm{ml}$ of ethanol (Merck) and $5 \mathrm{ml}$ of tetraethylorthosilicate (TEOS; Merck) were mixed in a beaker. After raising the temperature to $60^{\circ} \mathrm{C}$, under stirring, $100 \mu \mathrm{l}$ of $1 \mathrm{M} \mathrm{HCl}$ were added and incubation lasted for $50 \mathrm{~min}$. Then the solution was distributed into Terazaki style microplates $(10 \mu \mathrm{l} / \mathrm{well})$ and allowed to solidify for about $24 \mathrm{~h}$.

Antigen immobilization - The POS/PVA beads were transferred to 96-wells tissue culture plates and activated by incubation with $2.5 \% \mathrm{w} / \mathrm{v}$ glutaraldehyde $(100 \mu \mathrm{l})$, prepared in $0.1 \mathrm{M} \mathrm{H}_{2} \mathrm{SO}_{4}$, for $1 \mathrm{~h}$ at $25^{\circ} \mathrm{C}$. After washing with deionized water $(3 \times 200 \mu \mathrm{l})$, the beads were incubated overnight at $4^{\circ} \mathrm{C}$ with the antigen solution $(100 \mu 1)$, prepared in PBS. The beads containing the covalently immobilized antigen were then washed twice with PBS containing $0.05 \% \mathrm{w} / \mathrm{v}$ Tween 20 (PBST; Sigma), blocked with 3\% $\mathrm{w} / \mathrm{v}$ BSA (Sigma) in PBS at $25^{\circ} \mathrm{C}$ for $1 \mathrm{~h}$ and washed once with PBST.

ELISA - The antigen containing bead was incubated in $100 \mu \mathrm{l}$ of serum diluted in PBST containing $1 \% \mathrm{w} / \mathrm{v}$ $\mathrm{BSA}$ at $37^{\circ} \mathrm{C}$ for $40 \mathrm{~min}$, washed three times with PBST and then incubated in $100 \mu$ l of goat anti-human IgG conjugated to horseradish peroxidase (Sigma) at $37^{\circ} \mathrm{C}$ for 35 min. Afterwards, three washings with PBST were carried out. The substrate solution $(50 \mu 1), 3,3^{\prime}, 5,5^{\prime}$-tetramethylbenzidine liquid substrate system for ELISA (TMB; Sigma), was added and incubated at room temperature $\left(28^{\circ} \mathrm{C}\right)$ for $15 \mathrm{~min}$, in the dark. The reaction was stopped with $2 \mathrm{M} \mathrm{H}_{2} \mathrm{SO}_{4}(25 \mu \mathrm{l})$. The beads were removed and the optical density (OD) of the supernatant determined at 450 $\mathrm{nm}$ in ELISA reader (Bio-rad). A control experiment (blank) was carried out similarly, except for the use of PBS instead of serum sample.

The antigen concentration, conjugate and sera dilution for the procedure described above were determined as follows. An ELISA was carried out to establish the most convenient dilution of conjugate (from 2,500 to 80,000 times), using fixed values of antigen concentration and serum dilution. With the conjugate dilution set, the procedure was then repeated, this time varying the antigen concentration ( 5 to $0.156 \mu \mathrm{g} / \mathrm{ml}$ ). At last, the serum titration $(1: 100$ to $1: 1,600)$ was accomplished, using the antigen and conjugate dilutions pre-determined.

The results obtained through this method were compared to those obtained with the use of an ELISA plate (Dynatech M-129A) instead of the POS/PVA beads, the only change in the procedure being the replacement of PBS for bicarbonate buffer $\mathrm{pH} 9.6$ during antigen immobilization.

\section{RESULTS}

Sera from 45 children, attending a public school in Jaboatão, PE, Brazil, of which 21 were positive and 24 negative, were analyzed by ELISA using either conventional PVC-plate or POS/PVA bead as solid-phases. The results, in optical density (OD) values, are presented in the Figure. The mean ( \pm standard deviation) $\mathrm{OD}$ values found for the ELISA using negative and positive sera and POS/PVA bead and conventional PVC-plate were, respectively, $0.060 \pm 0.079 ; 0.714 \pm 0.158 ; 0.132 \pm 0.035$ and $0.275 \pm 0.092$. The values established for the negative and positive sera in both procedures showed to be statistically different according to the Mann-Whitney test $(\mathrm{p}<$ 0.05). Furthermore, the Wilcoxon test showed that the higher OD values registered for the POS/PVA bead procedure were statistically significant. The cut-off points for both procedures were established as the mean OD value of the negative sera plus two times the SD. They were 0.202 and 0.218 for the conventional PVC-plate and POS/ PVA bead based ELISA, respectively.

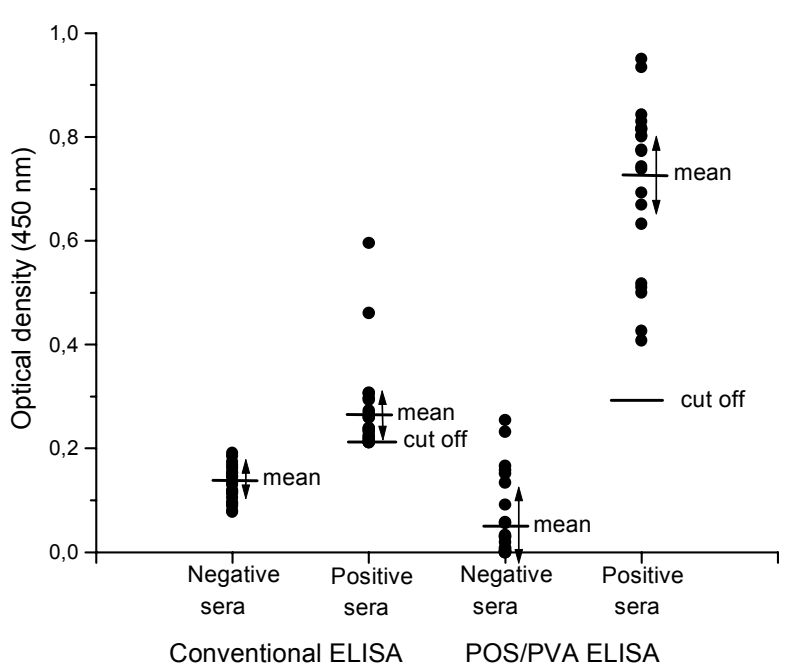

Comparison of the absorbance values obtained by conventional ELISA based on polyvinyl chloride (PVC) plates and ELISA based on POS-PVA beads. Sera from healthy and infected children were analyzed for the presence of anti-recombinant Toxocara canis antigen IgG. The absorbance reported is the mean of two determinations. The means (the arrow indicates the standard deviation interval) and cut-offs (the negative serum mean absorbance value plus two times its standard deviation) are indicated.

\section{DISCUSSION}

Excretory-secretory antigens derived from $T$. canis $\mathrm{L}_{2}$ maintained in defined medium in vitro have been commonly used for the immunodiagnosis of human toxocariasis (de Savigny et al. 1979, Lescano et al. 1998, Ajay et al. 2000). In the present work, recombinant antigen, presenting very low cross-reactivity with other helminthzoonosis (Yamasaki et al. 1998, 2000), was covalently immobilized onto polysiloxane/polyvinyl alcohol beads and used to detect human anti-Toxocara IgG.

Convenient parameters for carrying out the enzyme immunoassay were established as antigen concentration $31.2 \mathrm{ng} / \mathrm{bead}$, sera and conjugate diluted 200 and 10,000 times, respectively. It is worthwhile to notice that negligible unspecific human IgG is bound on the POS/PVA beads as it can be depicted by the low absorbance values observed in the blank assays (not shown).

Under those conditions, ELISA using POS/PVA beads as solid phase and recombinant $T$. canis antigen showed to be applicable to human IgG detection. Furthermore, the use of such support presents better results than conventional ELISA using PVC plates, as the difference between positive and negative sera mean optical densities is larger for this new glass based assay. 
In addition the performance of the POS/PVA bead as a matrix for immunodetection, its easy synthesis and the low cost are additional advantages for commercial application. More than 1,000 beads can be synthesized at a cost of US\$ 0.50 .

\section{REFERENCES}

Ajayi OO, Duhlinska DD, Agwale SM, Njoku M 2000. Frequency of human toxocariasis in Jos, Plateau State, Nigeria. Mem Inst Oswaldo Cruz 95: 147-149.

Barros AEL, Almeida AMP, Azevedo WM, Carvalho Jr LB 2002. Polysiloxane/PVA-glutaraldehyde hybrid composite as solid phase hybrid composite as solid phase for immunodetections by ELISA. Braz J Med Biol Res 35: 459463.

de Savigny DH, Voller A, Woodruff AW 1979. Toxocariasis: serological diagnosis by enzyme-immunoassay. $J$ Clin
Pathol 61: 781-782.

Lescano SAZ, Chieffi PP, Peres BA, Mello EO, Velarde CN, Salinas AA, Rojas CE 1998. Soil contamination and human infection by Toxocara sp. in the urban area of Lima, Peru. Mem Inst Oswaldo Cruz 93: 733-734.

Magnaval JF, Glickman LT, Dorchies P, Morassin B 2001. Highlights of human toxocariasis. Kor J Parasitol 39: 1-11.

Yamasaki H, Araki K, Lim PKC, Zasmy N, Mak JW, Taib R, Aoki T 2000. Development of a highly specific recombinant Toxocara canis second-stage larva excretory-secretory antigen for immunodiagnosis of human toxocariasis. JClin Microbiol 38: 1409-1413.

Yamasaki H, Taib R, Watanabe Y, Mak JW, Zasmy N, Araki K, Chooi LPK, Kita K, Aoki T 1998. Molecular characterization of a cDNA encoding an excretory-secretory antigen form Toxocara canis second stage larvae and its application to the immunodiagnosis of human toxocariasis. Parasitol Int 47: 171-181. 